\title{
A novel method for endoscopic ultrasound-guided pancreatic rendezvous with a microcatheter
}

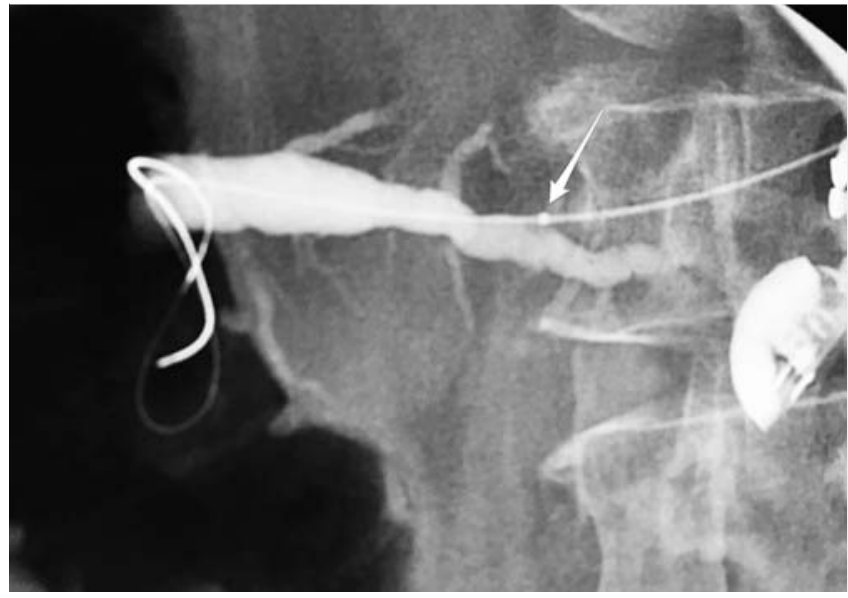
entering the pancreatic
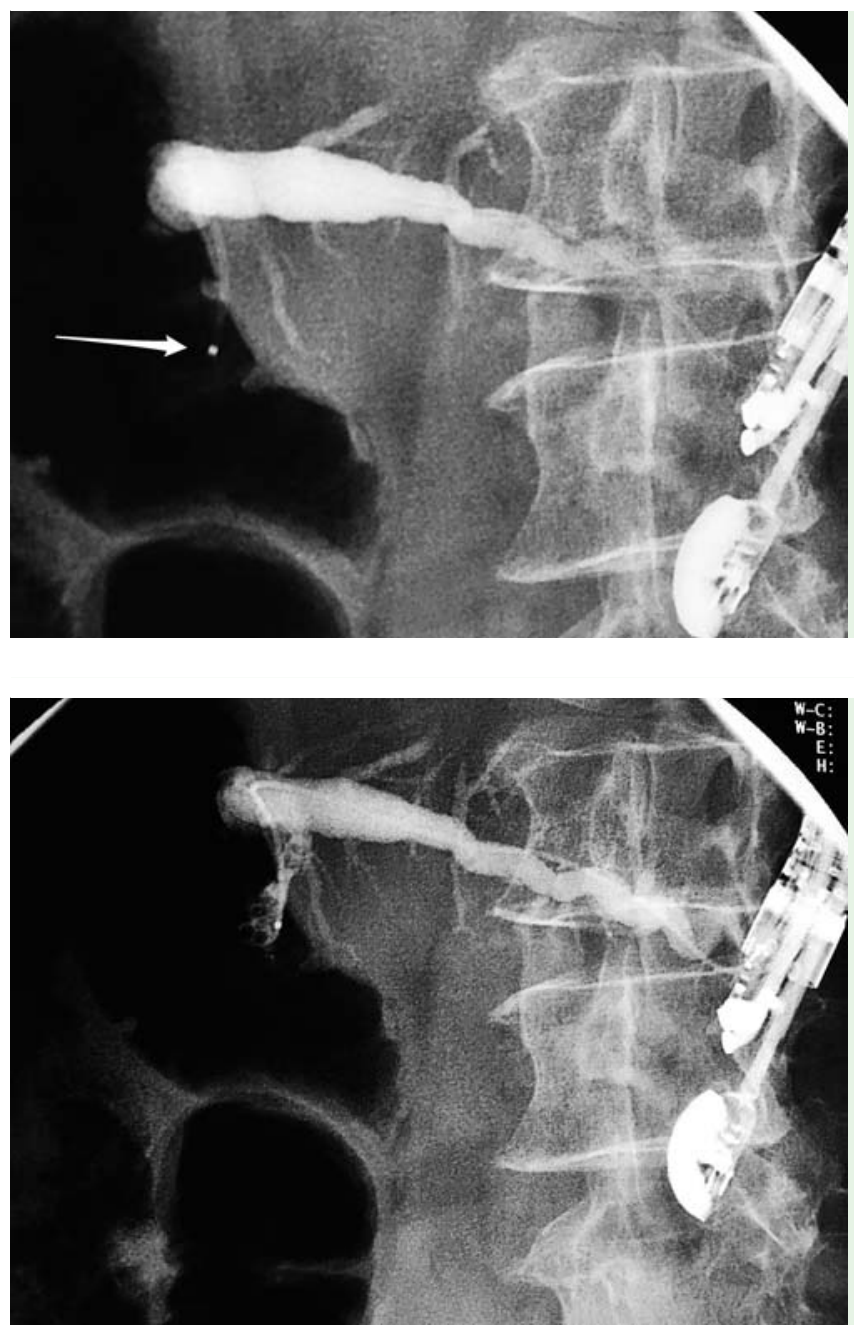

Fig. 1 The dorsal pancreatic duct is opacified and the guidewire looped in the prepapillary area of a 69-year-old woman with a fibrotic papillary stenosis preventing cannulation of the pancreatic duct. The microcatheter with the radiopaque marker (arrow) can be seen duct.

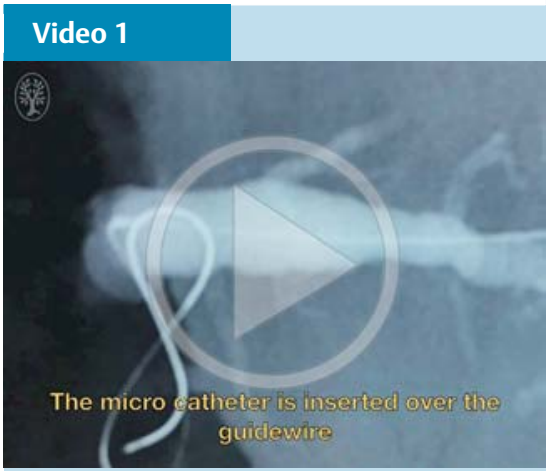

The complete process of rendezvous with the microcatheter. In the final image, the caliber of the microcatheter is compared with that of a 19-gauge needle.

Fig. 2 The microcatheter (arrow) has been advanced to the prepapillary area and the guidewire removed.

Fig. 3 Contrast is injected through the microcatheter to provide a complete map of the pancreatic duct.
A 69-year-old woman with chronic pancreatitis and recurrent pancreatic-type abdominal pain underwent computed tomography, which showed dilatation of the pancreatic duct. Subsequent endoscopic retrograde cholangiopancreatography revealed a fibrotic papillary stenosis preventing cannulation of the pancreatic duct.

Endoscopic ultrasound (EUS)-guided rendezvous was attempted ( $\bullet$ Video 1 ). The pancreatic duct was punctured with a 19-gauge needle (Expect; Boston Scientific, Natick, Massachusetts, USA). A 0.025-inch angulated-tip guidewire (VisiGlide; Olympus America, Center Valley, Pennsylvania, USA) was then advanced into the pancreatic duct but could not pass through the stenosis to the duodenal lumen. Because of the possible risk for fragmentation of the guidewire during manipulation, the needle was removed and a $150-\mathrm{cm}, 3-\mathrm{Fr}$ microcatheter (Renegade Hi-Flo; Boston Scientific) was inserted into the pancreatic duct over the guidewire ( $\bullet$ Fig. 1 ). The microcatheter was smoothly inserted and easily advanced to the prepapillary area, and the guidewire was removed ( $\bullet$ Fig.2). Contrast was injected to better define the cephalic duct ( Fig.3). A 0.025-inch straight-tip guidewire was inserted through the microcatheter ( $\bullet$ Fig.4) and after manipulation was advanced through the stenosis into the duodenum ( $\bullet$ Fig.5). The pancreatic rendezvous was complet- 


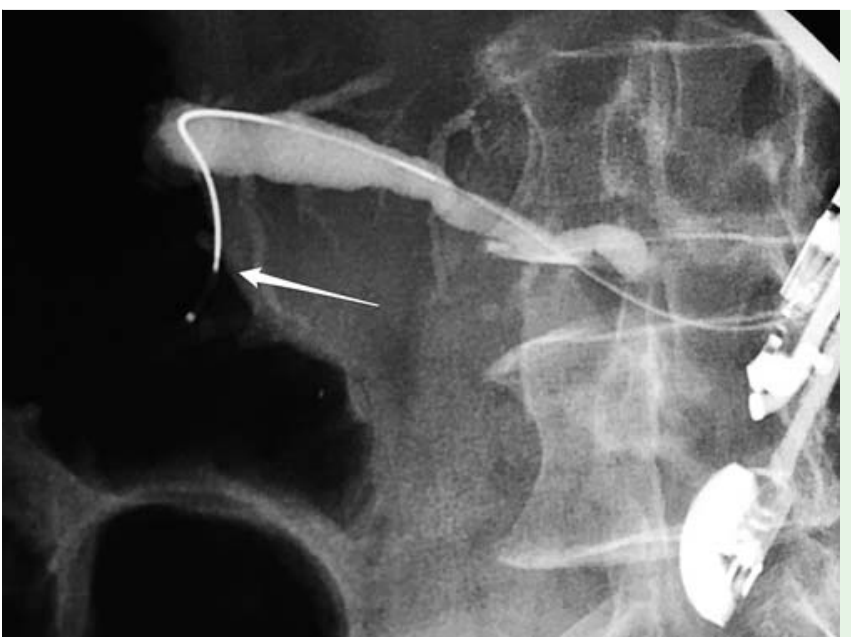

Fig. 4 A 0.025 -in straight-tip guidewire (arrow) is advanced through the microcatheter and comfortably manipulated.

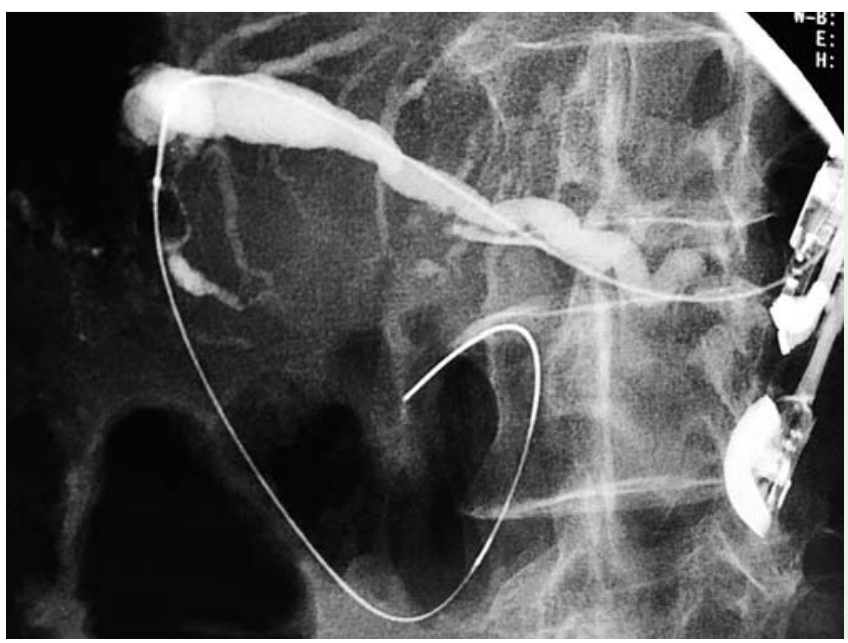

Fig. 5 With manipulation, the guidewire finally reaches the duodenal lumen through the stenosis, and the rendezvous procedure is completed.

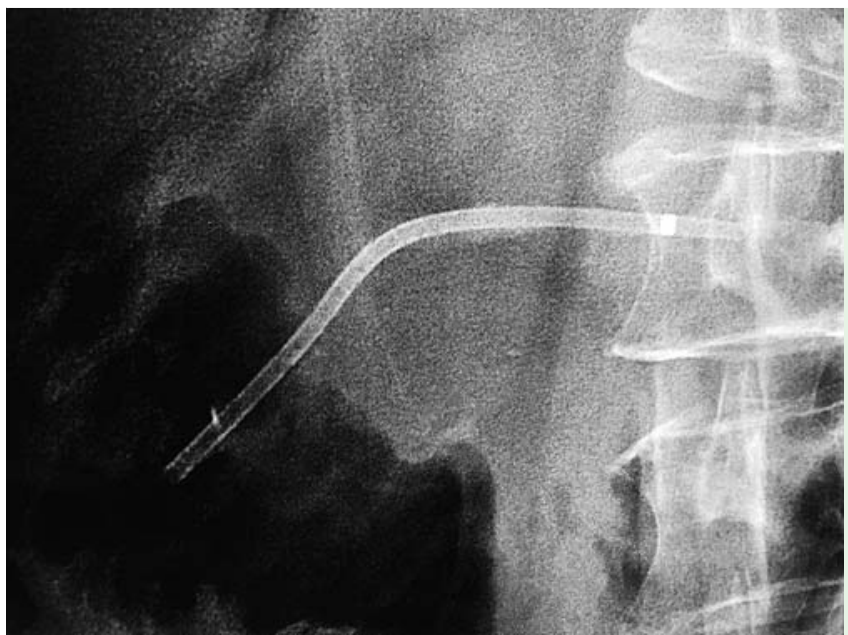

Fig. 6 A 7-Fr pancreatic stent is finally placed, and the patient is asymptomatic when discharged 24 hours later.

ed, and a 7-Fr pancreatic stent (Advanix; Boston Scientific) was placed ( $\bullet$ Fig. 6 ). The success rate for pancreatic rendezvous reaches only $50 \%$ in published series [1]. Manipulation of the guidewire, the most significant limiting factor [2], is hampered by the sharp needle grind, which can block and cut the tip of the guidewire. An enhanced protocol for biliary rendezvous with a 4-Fr catheter has been proposed [3]; however, this is the first report of EUS-guided rendezvous with a 3-Fr microcatheter. The microcatheter, taken from the interventional radiol- ogy armamentarium, is thinner than the 19-gauge needle, avoids dilation of the transmural track, and facilitates manipulation of the 0.025 -in guidewire, guidewire exchange, and contrast injection. These advantages can improve the success rate of EUS-guided rendezvous.

Endoscopy_UCTN_Code_TTT_1AS_2AD

Competing interests: None

\section{Juan Vila ${ }^{1}$, Carlos Huertas², Bruno Gonçalves ${ }^{3}$, Alba Cebrián', David Ruiz-Clavijo', Belén González de la Higuera', Carlos Prieto ${ }^{1}$}

${ }^{1}$ Endoscopy Unit, Gastroenterology Department, Complejo Hospitalario de Navarra, Pamplona, Spain

2 Gastroenterology Department, Hospital Universitario Josep Trueta, Gerona, Spain ${ }^{3}$ Gastroenterology Department, Hospital de Braga, Braga, Portugal

\section{References}

1 Takikawa T, Kanno A, Masamune A et al. Pancreatic duct drainage using EUS-guided rendezvous technique for stenotic pancreaticojejunostomy. World J Gastroenterol 2013; 19: $5182-5186$

2 Vila JJ, Pérez-Miranda M, Vazquez-Sequeiros $E$ et al. Initial experience with EUS-guided cholangiopancreatography for biliary and pancreatic duct drainage: a Spanish national survey. Gastrointest Endosc 2012; 76 : $1133-1141$

3 Park do H, Jeong SU, Lee BU et al. Prospective evaluation of a treatment algorithm with enhanced guidewire manipulation protocol for EUS-guided biliary drainage after failed ERCP (with video). Gastrointest Endosc 2013; 78: 91 - 101

\section{Bibliography}

Dol http://dx.doi.org/

10.1055/s-0034-1393373

Endoscopy 2015; 47: E575-E576

(C) Georg Thieme Verlag KG

Stuttgart · New York

ISSN 0013-726X

\section{Corresponding author} Juan J. Vila, MD

Endoscopy Unit, Gastroenterology Department Complejo Hospitalario de Navarra

$\mathrm{C} /$ de Irunlarrea 3

31008 Pamplona

Navarra

Spain

Fax: +34-848-422303

juanjvila@gmail.com 